\title{
UHPLC-MS/MS profiling of histidine and bile acid metabolism in human gastric fluid for diagnosis of gastric diseases
}

\author{
Wonwoong Lee ${ }^{1 \dagger}$, Jinhee Um ${ }^{1 \dagger}$, Keon-hee Ko ${ }^{1}$, Yong Chan Lee ${ }^{2}$, Bong Chul Chung ${ }^{3^{*}}$ and Jongki Hong ${ }^{*^{*}}$
}

\begin{abstract}
Bile acids (BAs) are synthesized in the liver and can mediate homeostasis and various metabolism processes in the human body. Their levels in the gastrointestinal tract are closely related to various gastrointestinal diseases. In particular, farnesoid $\mathrm{X}$ receptor activated by free BAs is associated with overexpression of histidine decarboxylase in tumorigenesis. Therefore, comprehensive profiling of histamine (HIST), histidine (His), and BAs in biological samples can provide insight into the pathological mechanisms of gastrointestinal diseases. However, development of an analytical platform to profile HIST, His, and BAs in biological samples has several challenges such as highly different polarities between acidic and basic targets, low physiological concentrations of analytes, and high matrix interference of biological samples. In this study, an ultra-high performance liquid chromatography-tandem mass spectrometry (UHPLC-MS/MS) method combined with serial derivatization was developed to simultaneously determine HIST, His, and 5 BAs (cholic acid, deoxycholic acid, chenodeoxycholic acid, ursodeoxycholic acid, and lithocholic acid) in human gastric fluid. In serial derivatization, benzoyl chloride (BzCl) and $\mathrm{N}, \mathrm{N}$ dimethylethylenediamine (DMED) were used to selectively derivatize amino and carboxyl groups of analytes, respectively. After serial derivatization, all target derivatives were determined using a reverse-phase C18 LC column and positive multiple reaction monitoring (MRM) mode, with reasonable chromatographic separation and sensitive MS detection. To accurately quantify target metabolites, 7 stable isotope-labeled internal standards were used. The MS/MS spectra of DMED and Bz derivatives exhibited specific fragments via loss of a neutral molecule (dimethylamine; $45 \mathrm{Da}$ ) and inductive cleavage (benzoyl; $\mathrm{m} / \mathrm{z}$ 105) from protonated molecules, enabling selection of appropriate MRM transition ions for selective and sensitive detection. The developed method was validated with respect to limits of detection and quantification, linearity, precision, accuracy, stability, and matrix effect. The established method was successfully applied to human gastric fluid samples. This method provides reliable quantification of HIST, His, and BAs in human gastric fluid and will be helpful to understand pathophysiological mechanisms of gastric diseases.
\end{abstract}

Keywords: Bile acids, Histidine, Histamine, UHPLC-MS/MS-MRM, Profiling analysis, Serial derivatization, Human gastric fluid, Gastric cancer

\footnotetext{
* Correspondence: bcc0319@kist.re.kr; jhong@khu.ac.kr

${ }^{+}$Wonwoong Lee and Jinhee Um contributed equally to this work.

${ }^{3}$ Molecular Recognition Research Center, Korea Institute of Science and Technology, Seoul 02792, Republic of Korea

${ }^{1}$ College of Pharmacy, Kyung Hee University, Seoul 02447, Republic of Korea

Full list of author information is available at the end of the article
}

\section{Springer Open}

(c) The Author(s). 2020 Open Access This article is licensed under a Creative Commons Attribution 4.0 International License, which permits use, sharing, adaptation, distribution and reproduction in any medium or format, as long as you give appropriate credit to the original author(s) and the source, provide a link to the Creative Commons licence, and indicate if changes were made. The images or other third party material in this article are included in the article's Creative Commons licence, unless indicated otherwise in a credit line to the material. If material is not included in the article's Creative Commons licence and your intended use is not permitted by statutory regulation or exceeds the permitted use, you will need to obtain permission directly from the copyright holder. To view a copy of this licence, visit http://creativecommons.org/licenses/by/4.0/. 


\section{Introduction}

Bile acids (BAs), which are metabolized in the cholesterol cascade in the human liver and stored in the gallbladder, are important regulators of homeostasis in the human body (Bernstein et al. 2009). In particular, as a BA receptor, farnesoid X receptor (FXR) plays an intriguing role in inflammation, metabolic diseases, and cancer (Gadaleta et al. 2015; Wan and Sheng 2018), and is activated by only free BAs (Makishima et al. 1999; Parks et al. 1999). Furthermore, activated FXR by free BAs can induce overexpression of histidine decarboxylase (HDC) that converts histidine (His) into histamine (HIST); this pathway might be correlated with gastric cancer (Falus et al. 2011; Ku et al. 2014). Therefore, the profiling of HIST, His, and BAs is crucial to understand the mechanism of stomach carcinogenesis.

Gastric fluid is produced by numerous cells (such as parietal, chief, mucus-secreting, and hormone-secreting cells) in the gastric glands and is composed of water, electrolytes, hydrochloric acid, various enzymes, mucus, and intrinsic factors (Gropper et al. 2005). Moreover, normal human gastric fluid is a translucent liquid and may include salivary contents via swallowing, bile by duodenogastric reflux, and mediators of inflammation or blood from damaged stomach walls (Lu et al. 2010). In addition to major body fluids such as blood and urine, gastric fluid can contain substantial information related to metabolic state and can reflect physiological conditions especially in the stomach (Deng et al. 2012). Hence, it is thought that biomarkers for various gastrointestinal diseases could exist in gastric fluid.

From determination of gastric acidity to sophisticated instrumental analysis, laboratory analysis of gastric fluid has been implemented by various means. In particular, liquid chromatography-mass spectrometry (LC-MS) has been employed to identify and quantify biochemicals in gastric fluid due to its high sensitivity and selectivity. To gather important pathophysiological information about gastric cancer, several LC-MS methods have been developed to determine metabolites in gastric fluid. For example, to discover biomarkers for gastric cancer diagnosis, Choi et al. (Choi et al. 2016) developed an LC-MS/MS method to determine tryptophan and its 7 metabolites in serum and gastric fluid from patients with gastritis or gastric cancer. Moreover, for discovery of diagnostic biomarkers for early gastric cancer, LC-MS/ MS methods were developed to detect free and aromatic amino acids in gastric fluid samples (Liu et al. 2018; Liu et al. 2017). Similarly, Deng et al. (Deng et al. 2011) developed an LC-MS method to identify and validate fluorescence detection of aromatic amino acids in gastric fluid.

Although several advanced LC-MS methods have been introduced to determine various types of metabolites, these methods are still difficult to simultaneously determine acid and basic targets in biological samples. This is because these target analytes consist of biochemicals with different physicochemical properties (such as polar and basic His and HIST and relatively non-polar and acidic BAs). Most of all, common LC column could not be properly employed to simultaneously separate HIST, His, and free BAs. For instance, although a hydrophilic interaction LC column has been successfully employed to separate polar neurotransmitters including HIST and His (Tufi et al. 2015), it could be inappropriate to directly apply non-polar BAs. Reversed-phase (RP) LC column is not suitable for obtaining high peak capacity of His and HIST, while it was effectively used to separate BAs (Yin et al. 2017). Meanwhile, derivatization enables sufficient chromatographic separation as well as sensitive and selective MS detection of acidic and basic targets via tuning of physicochemical properties of analytes. Furthermore, serial derivatization can minimize timeconsuming and laborious process of individual derivatization for multifunctional groups on analytes.

In this study, we developed an ultra-high performance (UHP) LC-MS/MS method combined with serial derivatization of primary amines and carboxylic acids on analytes to simultaneously profile HIST, His, and free BAs in gastric fluid. This developed analytical method was successfully used to analyze human gastric fluid samples from patients with chronic superficial gastritis (CSG). This method is expected to be a potent analytical platform to provide reliable quantification of $5 \mathrm{BAs}$, His, and HIST in biological samples, and it will be helpful in the understanding of pathophysiological mechanisms of gastric cancer and diagnosis of gastric diseases.

\section{Experimental}

\section{Chemicals and materials}

All authentic standards and chemicals were analytical grade or better. Methanol $(\mathrm{MeOH})$, acetonitrile $(\mathrm{ACN})$ and formic acid were purchased from Honeywell (NJ, USA). De-ionized water (DW) was produced using a Millipore Direct-Q3 purification system from Millipore Corporation (Billerica, MA, USA). Simulated gastric fluid (SGF) was obtained from Thermo Fisher Scientific (Waltham, MA, USA). Unconjugated bile acids (cholic acid (CA), deoxycholic acid (DCA), chenodeoxycholic acid (CDCA), ursodeoxycholic acid (UDCA), lithocholic acid (LCA)), L-histidine (His), His- ${ }^{13} \mathrm{C}_{6}$ hydrochloride monohydrate, histamine (HIST), benzoyl chloride (BzCl), sodium carbonate, $\mathrm{N}, \mathrm{N}$-dimethylethylenediamine (DMED), trimethylamine (TEA), and 2-chloro-1-methylpyridinium iodide (CMPI) were purchased from Sigma-Aldrich (St. Louis, MO, USA). CA- $\mathrm{d}_{4}$, DCA- $\mathrm{d}_{4}$, CDCA- $\mathrm{d}_{4}$, UDCA- $\mathrm{d}_{4}$, LCA- $\mathrm{d}_{4}$, and HIST- $\mathrm{d}_{4}$ were obtained from CDN Isotopes (Pointe-Claire, Quebec, Canada). 


\section{Human gastric fluid}

Twenty gastric fluid samples from patients with CSG ( $n$ = 20) were collected using gastroduodenoscopy at Severance Hospital, Seoul, Korea. These patients were categorized by Helicobacter pylori IgG testing and gastrin level to detect $H$. pylori infection. Consequently, 5 CSG gastric fluid samples with and 15 without $H$. pylori infection were collected. Collected gastric fluid samples were immediately frozen in liquid nitrogen and stored at $-80^{\circ} \mathrm{C}$ until analysis. This study was approved by the ethical committee of Severance Hospital (4-2013-0880).

\section{Sample clean-up and extraction and serial derivatization} Fifty microliters of gastric fluid sample was thawed at room temperature, followed by transfer into a $1.5-\mathrm{mL}$ centrifuge tube spiked with $50 \mu \mathrm{L}$ of internal standard solution $\left(2 \mu \mathrm{g} / \mathrm{mL}\right.$ of His- ${ }^{13} \mathrm{C}_{6}$ and $200 \mathrm{ng} / \mathrm{mL}$ of a mixture of HIST-d4, CA- $\mathrm{d}_{4}$, DCA- $\mathrm{d}_{4}$, CDCA- $\mathrm{d}_{4}$, UDCA- $\mathrm{d}_{4}$, and LCA- $\mathrm{d}_{4}$ ). Fifty microliters of ice-cold ACN was added to the gastric fluid sample, which was then vigorously shaken for $1 \mathrm{~min}$ and centrifuged at 14,000 rpm for 10 min. After centrifugation, $100 \mu \mathrm{L}$ of supernatant was transferred to a glass vial for derivatization.

For serial derivatization, benzoylation using benzoyl chloride was performed to derivatize the primary amino group on His and HIST, followed by an amide reaction using DMED for the carboxyl group. To adjust the sample $\mathrm{pH}, 2 \mu \mathrm{L}$ of $1 \mathrm{M}$ TEA in $\mathrm{ACN}$, as an $\mathrm{HCl}$ acceptor, was added to the supernatant. Next, $50 \mu \mathrm{L}$ of $100 \mathrm{mM}$ sodium carbonate buffer and $50 \mu \mathrm{L}$ of $2 \%$ benzoyl chloride in ACN were added to the vial. The benzoylation reaction was conducted at room temperature for 1 min, followed by addition of $10 \mu \mathrm{L}$ of $8 \mathrm{mM}$ TEA in $\mathrm{ACN}$ for a quenching reaction. Fifty microliters of 100 $\mathrm{mM}$ CMPI in ACN as condensation reagent and $20 \mu \mathrm{L}$ of $250 \mathrm{mM}$ DMED in ACN were added to sample aliquots. The mixed sample was heated at $60^{\circ} \mathrm{C}$ for $40 \mathrm{~min}$, followed by transfer to a deep freezer for $10 \mathrm{~min}$ for the quenching reaction. The sample solution was then dried under a gentle stream of nitrogen gas. The dried residue was reconstituted in $50 \mu \mathrm{L}$ of mixture of $0.01 \%$ formic acid in DW and ACN $(95: 5, v / v)$, followed by injection into the LC-MS/MS system. The overall analytical platform including sample preparation procedures is depicted in Fig. 1.

\section{UHPLC-MS/MS-MRM}

Chromatographic separations were performed on a Waters ACQUITY UPLC BEH C18 column $(100 \times 2.1 \mathrm{~mm}$, $1.7 \mu \mathrm{m}$ ) using a Waters UPLC $\mathrm{H}$ class system (Waters Corporation, Milford, MA, USA). Mobile phases A and $\mathrm{B}$ were $0.01 \%$ formic acid in water and $\mathrm{ACN}$, respectively. Gradient elution was initiated with $5 \%$ of mobile phase $B$ for $0.0-0.5 \mathrm{~min}, 5-35 \%$ of $B$ for $0.5-1.0 \mathrm{~min}$, $35-30 \%$ of B for $1.0-4.0 \mathrm{~min}, 30 \%$ of B for $4.0-5.0 \mathrm{~min}$, $30-40 \%$ of B for $5.0-6.0 \mathrm{~min}, 40-45 \%$ of B for $6.0-8.0$ min, $45-70 \%$ of B for $8.0-11.5 \mathrm{~min}, 70-100 \%$ of $B$ for $11.5-12.0 \mathrm{~min}, 100 \%$ of $\mathrm{B}$ for $12.0-13.0 \mathrm{~min}$, and $5 \%$ of B for 13.1-15.0 min for re-equilibration. Injection volume of samples and flow rate were set at $10 \mu \mathrm{L}$ and
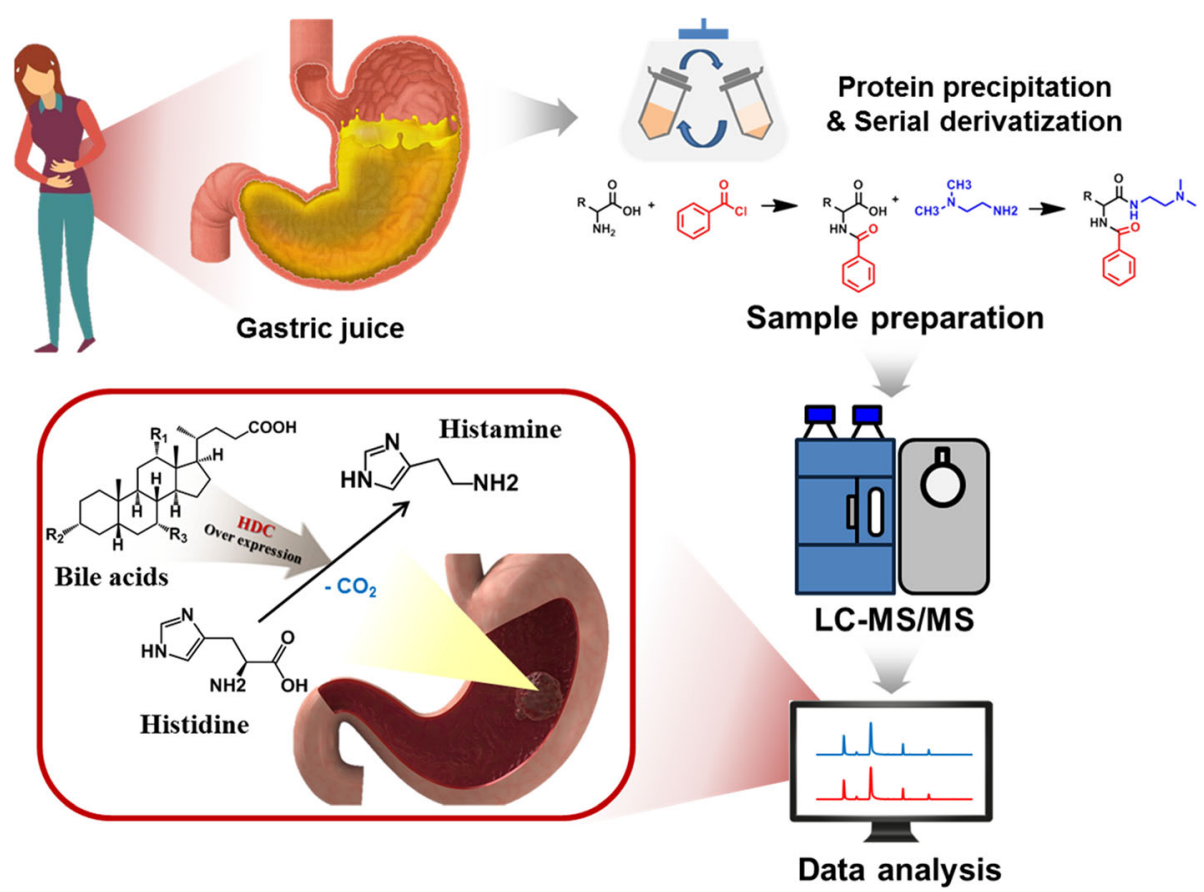

Fig. 1 Analytical procedure for profiling of HIST, His, and BAs in gastric fluid 
$300 \mu \mathrm{L} / \mathrm{min}$, respectively. Applied chromatographic separation conditions were modified from a previous report (Yin et al. 2017).

Each target metabolite was analyzed using an API 3200 triple quadrupole mass spectrometer system (Applied Biosystems Inc., Foster City, CA, USA). All analytes were detected by Turbo $\mathrm{V}$ electrospray ionization (ESI) in positive ion multiple reaction monitoring (MRM) mode. Mass spectrometric parameters were set as follows: ion spray voltage at $5200 \mathrm{~V}$, curtain gas at 20 psi, nebulizer gas at $50 \mathrm{psi}$, auxiliary gas at $50 \mathrm{psi}$, temperature at $500{ }^{\circ} \mathrm{C}$, and collision-activated dissociation gas at 5 . Nitrogen gas was used for ion source nebulizing and tandem MS collision. Quantifier and qualifier MRM transition ions were selected as the most abundant product ion and the characteristic fragment ion, respectively. Optimized MRM conditions are shown in Suppl. Table 1. Data acquisition and analysis were achieved with the Analyst Software 1.5.2 by Applied Biosystems.

\section{Method validation}

The developed UHPLC-MS/MS method combined with serial derivatization was validated according to the bioanalytical validation method provided by the US Food and Drug Administration (FDA) (U.S. Department of Health and Human Services Food and Drug Administration 2018). Linearity was evaluated for each analyte over different concentration ranges. The limits of detection (LODs) were defined as concentration levels with a signal-to-noise ratio $>3$. The limits of quantification (LOQs) were determined as the lowest concentration levels with acceptable precision and accuracy. The precision and accuracy were validated with quality control samples at high, medium, and low concentration levels in 6 replicates within 1 day and on 6 different days. Recoveries of the developed method were calculated as [(spiked SGF)/(standard solutions) $\times 100(\%)]$. The matrix effect of gastric fluid samples was investigated using 7 corresponding stable isotope-labeled (SIL) internal standards and calculated as [(spiked gastric fluid)/ (standard solution) $\times 100(\%)]$. The stability tests of each compound were assessed using spiked samples at three concentration levels under different storage and working conditions (room temperature, $4{ }^{\circ} \mathrm{C}$, and 3 freeze-thaw cycles), before processing.

\section{Results and discussion}

\section{Optimization of serial derivatization} Serial derivatization

When developing an analytical method to simultaneously determine BAs, His, and HIST in gastric fluid, there are several challenges such as high matrix effect, low physiological levels of metabolome, and different chemical properties between analytes. Among them, the different chemical properties among basic HIST, amphiphilic His, and acidic BAs make it difficult to develop a single analytical platform. Furthermore, His and HIST are difficult to hydrophobically interact with RPLC column due to their polar nature, and rigid steroidal structures of BAs seldom produce abundant characteristic fragments. For these reasons, it is necessary to apply a derivatization method to tune the physicochemical characteristics of analytes. As a representative instance, derivatization of amino groups using benzoyl or dansyl chloride has been widely used to increase sensitivity and hydrophobicity (Lu et al. 2016; Malec et al. 2017). For carboxyl groups, derivatization using $\mathrm{N}, \mathrm{N}$-dimethylethylenediamine (DMED) and esterification of carboxylic acids have been employed to improve proton affinity under positive ion ESI mode (Gao et al. 2009; He et al. 2019; Lee et al. 2017; Zhu et al. 2016).

Although several advanced derivatization processes have been developed, it is difficult to simultaneously derivatize different functional groups. Since derivatization procedures for primary amino and carboxyl groups demand different conditions, 2 distinct derivatization methods should be employed, in series or in parallel (Huang et al. 2019; Lee et al. 2020). A parallel derivatization approach to 4 different functional groups (carboxyl, carbonyl, amine, and thiol) has been conducted to profile the comprehensive fecal metabolome of mice (Yuan et al. 2018). On the other hand, using dimethylaminoacetyl chloride and DMED, a serial derivatization approach for amino, hydroxyl, and carboxyl groups on organic acids and amino acids has been employed to simultaneously detect all components of the target metabolome in human tissue samples (Huang et al. 2018b; Huang et al. 2018a). In particular, the latter technique enables a single analysis through use of a one-pot derivatization system. By taking advantage of clinical applications requiring high throughput, serial derivatization enabling a one-pot derivatization system was adopted (Suppl. Fig. 1).

\section{Decision of derivatization reaction and order}

$\mathrm{BzCl}$ as a derivatization reagent was used to increase retention of His and HIST on RPLC through protection of their primary amino groups. Benzoylation has been extensively employed to increase sensitivity and selectivity of polar amine and phenolic metabolites in biological samples (Song et al. 2012; Wong et al. 2016; Zheng et al. 2012). At the same time, the DMED derivatization reagent was chosen to selectively label carboxyl groups on His and BAs. Through DMED amidation, His and BAs could be not only remarkably improved detection sensitivity in ESI positive ion mode, but also improved 
selectivity by producing specific MRM transition ions especially for BAs, as indicated in Suppl. Table 1.

As a serial derivatization method involves sequential reactions with amino and carboxyl groups, the derivatization order had to be considered. In the preliminary experiment, benzoylation before amidation was preferred (Suppl. Fig. 2). This may be because, when carboxyl groups are being derivatized, they can react with amino groups on the analytes rather than the amine reagents (Huang et al. 2019). Therefore, derivatization of amine analytes should precede before carboxylic acid derivatization, or excessive amine reagent should be used. Based on individually optimized conditions of benzoylation and amidation in previous reports (Wong et al. 2016; Zhu et al. 2015), several conditions of a serial derivatization method were modified and developed.

\section{Optimization of derivatization conditions}

Since benzoylation was performed as a first derivatization step, base hydrolysis of $\mathrm{Bz}$ derivatives of His and HIST was a concern due to the alkaline environment during DMED derivatization. To address this issue, concentrations of derivatization reagents were optimized. To obtain the highest possible DMED derivatization yields, DMED concentrations ranging from 50 to 300 $\mathrm{mM}$ were tested, maintaining a molar ratio of 1:1 between DMED and condensation reagent (CMPI). As shown in Fig. 2a, the reaction yields of most DMED derivatives were highest at a DMED concentration of 250 $\mathrm{mM}$, while Bz derivatives of HIST were drastically decreased at DMED concentrations higher than $100 \mathrm{mM}$. As a catalyst, TEA concentrations from 0 to $10 \mathrm{mM}$ were also investigated. The reaction yields of all DMED derivatives for BAs were increased up to $8 \mathrm{mM}$ of TEA (Fig. 2b). Using the different physiological concentrations for HIST, His, and BAs, derivatization conditions were optimized according to highest derivatization yields for BAs. Compared with previously optimized DMED derivatization procedures ( $\mathrm{He}$ et al. 2019; Zhu et al.
2016; Zhu et al. 2015), excessive concentrations of derivatization reagents were necessary to optimize the DMED derivatization yields. After benzoylation, excessive reagents to derivatize the primary amine on His and HIST were remained during the second derivatization step. Therefore, it was speculated that residual reagent may have reacted with DMED derivatization reagents. Under optimized derivatization conditions, the efficiency of a serial derivatization method for BAs was higher than that of individual DMED derivatization (Fig. 3).

Although the reaction conditions of a serial derivatization were optimized, it is difficult to directly apply the method to gastric fluid due to its inherent physiological characteristics. In gastric fluid, hydrochloric acids $(\mathrm{HCl})$ secreted from parietal cells in gastric glands are ranges in concentration from 150 to 160 $\mathrm{mM}$, producing a low $\mathrm{pH}$ (Gropper et al. 2005; Smith 2003). These high $\mathrm{HCl}$ concentrations could inhibit reactivity between derivatization reagents and analytes. Moreover, benzoylation of primary amines should be conducted at $\mathrm{pH}>8$, since the reactivity of amine is dependent on its deprotonization (Huang et al. 2019). To counter the hindrance effect of $\mathrm{HCl}$, addition of TEA, as an $\mathrm{HCl}$ acceptor, was investigated. The efficiency of formation of $\mathrm{Bz}$ derivatives with $0-50 \mathrm{mM}$ of TEA was calculated by relative peak area. Before the benzoylation reaction, TEA was added to SGF, which has a chemical composition and concentration similar to human gastric fluid except for endogenous metabolites. When $10 \mathrm{mM}$ of TEA was added, benzoylation yields of His and HIST were higher than those in SGF along (Fig. 4). However, benzoylation yields decreased at TEA concentrations over $20 \mathrm{mM}$. It was speculated that addition of $10 \mathrm{mM}$ of TEA was sufficient for high benzoylation of His and HIST, since additional sodium carbonate buffer was added for the benzoylation reaction. Therefore, in this study, $10 \mathrm{mM}$ of TEA was employed as an $\mathrm{HCl}$ acceptor for serial derivatization in gastric fluid.

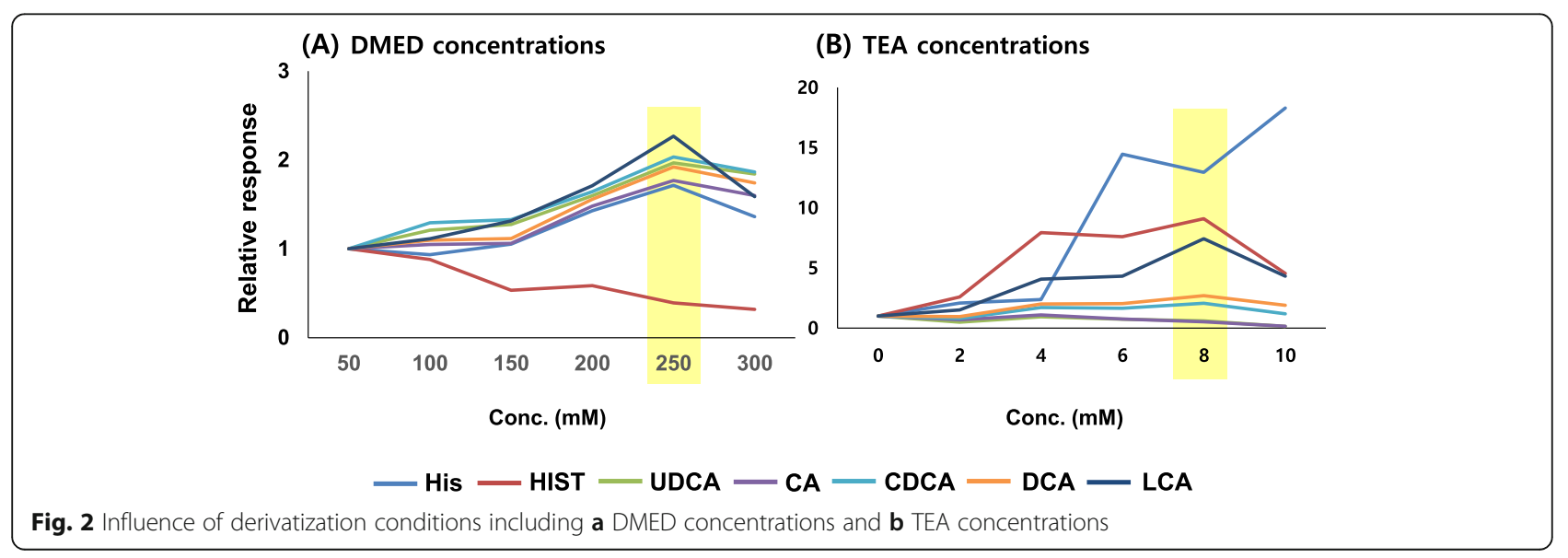




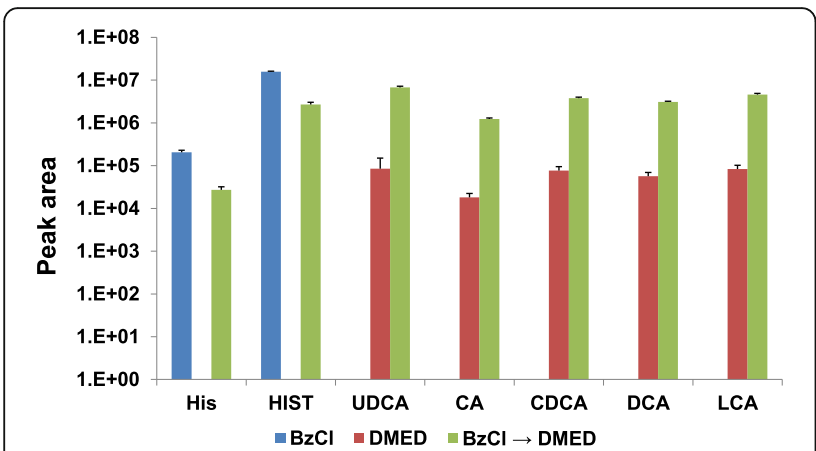

Fig. 3 Comparison of individual and serial derivatization

After application of a developed protocol, the derivatization efficiencies for HIST, His, and BAs were evaluated by relative response of derivatives and non-derivatized forms. As shown in Suppl. Table 2, while overall yields for DMED derivatives of BAs were higher than 95.27\%, derivatization yields of HIST-Bz derivative and His-Bz DMED derivatives were $78.23 \%$ and $65.51 \%$, respectively. No by-product for HIST and BAs was found after serial derivatization reactions. During serial derivatization of His, side products such as His-Bz and His-DMED were found at small amounts below 5\% level. However, relative responses of two side products were negligible. Therefore, it was speculated that $\mathrm{Bz}$ derivatives of $\mathrm{His}$ and HIST formed by first step derivatization were gradually degraded throughout DMED derivatization procedure. Nevertheless, this derivatization method enabled not only highly reproducible reactions for all derivatives of target analytes, but also sensitive detection of all target metabolites in gastric fluids.

\section{UHPLC-MS/MS}

Without derivatization, His and HIST are eluted at/near void volume due to their highly polar imidazole ring and primary amine when they are separated on a traditional

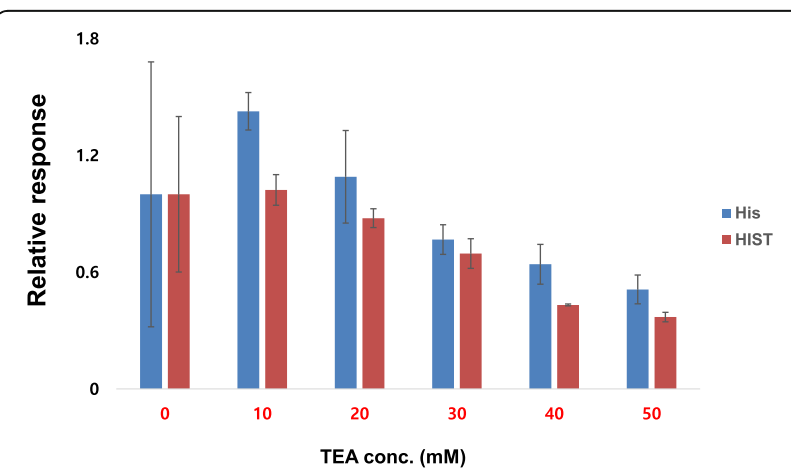

Fig. 4 Comparison of derivatization efficiencies in SGF: influence of $\mathrm{HCl}$ acceptor (TEA)
RPLC column (Gornischeff et al. 2018). In analysis of biological samples, matrix-related ion suppression is mainly observed in the front region of chromatograms, which corresponds to the void volume of the column (Annesley 2003; Dams et al. 2003). Moreover, polar and small molecules such as His and HIST are susceptible to ion suppression, which can affect quantitative profiling (Annesley 2003). To minimize ion suppression, it is necessary to apply the derivatization method to increase of hydrophobicity and molecular weight. Therefore, after application of the developed derivatization method, derivatives of His and HIST were well retained and separated on an RP C18 column (Suppl. Fig. 2(B) and 3).

On the other hand, although non-polar BAs are well retained on a RPLC column, BAs are difficult to separate on a conventional column. This is because BAs have a similar chemical structure (steroid nucleus), and some BAs are isomers. For this reason, UDCA:CA and CDCA: DCA were still not separated after derivatization (Suppl. Fig. 2(B)). Furthermore, CDCA and DCA exhibited similar MS/MS fragmentation patterns and shared MRM transitions. Therefore, chromatographic separation conditions had to be optimized to separate the overall analytes, especially CDCA and DCA. Fortunately, an extraordinary gradient elution program to separate unconjugated BAs was reported (Yin et al. 2017), and in this study, an LC method was optimized and modified based on that previous report. Under optimized chromatographic conditions, without support of ion-pairing reagents or hydrophilic interaction LC, all derivatives including polar His and HIST exhibited reasonable separation on an RPLC column using conventional mobile phases: mobile phase A, de-ionized water with $0.01 \%$ formic acid additive; mobile phase B, acetonitrile (Suppl. Fig. 3). In particular, derivatives of His and HIST were well retained on the RPLC column, avoiding elution in the void volume.

In addition, since BAs have a rigid steroid nucleus and carboxylic acid side chain, neither MRM detection using characteristic fragment ions nor determination of protonated molecules $\left([\mathrm{M}+\mathrm{H}]^{+}\right)$in positive ion mode is easy to perform (García-Cañaveras et al. 2012). Similarly, to apply positive ion MRM detection, it is necessary to introduce an appropriate derivatization method providing characteristic MRM transition ions and the high proton affinity of BAs. After application of the developed derivatization method, the DMED derivatives of BAs and the derivatives of HIST and His showed improved detection sensitivity and selectivity in positive ion MRM mode since both benzoylation and DMED amidation can produce characteristic fragment ions (benzoyl fragments; $m / z 105$ ) and neutral loss fragments (dimethylamine neutral fragments; $45 \mathrm{Da}$ ) (Wong et al. 2016; Zhu et al. 2016). Therefore, the derivatization method 
supported development of a sensitive and selective MRM detection method for quantification and/or qualification (Suppl. Table 1). Based on reasonable LC separations and selective MRM determination, the analytical method could be used to quantitatively profile all target analytes in gastric fluid.

\section{Method validation}

Under optimized instrumental and derivatization conditions, the developed analytical method was evaluated by the bioanalytical validation method provided by the US FDA (U.S. Department of Health and Human Services Food and Drug Administration 2018). The validation results for the analysis of SGF spiked with authentic chemical standards were corrected using the corresponding 7 SIL internal standards and are presented in Table 1. Calibration curves were constructed within different concentration ranges through analysis of 6 replicate samples. The determination coefficients for linearity $\left(r^{2}\right)$ were above 0.9938 for all investigated calibration curves, indicating that they were linear over dynamic ranges. The LODs, which were determined by the signal-tonoise ratios for all target analytes higher than 3, ranged from 2.32 to $182.97 \mathrm{ng} / \mathrm{mL}$. The LOQs were determined as the lowest calibration concentrations detected with acceptable precision (2.87-14.29\%) and accuracy (92.26$116.75 \%$ ) and were in the range of $25-250 \mathrm{ng} / \mathrm{mL}$. The developed method showed sufficient detection sensitivity for overall analytes of interest to enable trace-level profiling of gastric fluid samples with diverse physiological conditions. Precision and accuracy were investigated by analyzing different samples with low, medium, and high concentrations of analytes. Overall precision and accuracy of all target analytes were $1.89-13.04 \%$ and 87.34-113.31\%, respectively. As shown in Table 1, the recoveries of all targets ranged from 85.75 to $107.07 \%$. The stability and matrix effect of HIST, His, and BAs are summarized in Table 2 . The stability for all analytes ranged from $74.06-105.73 \%$ at room temperature and $75.79-109.64 \%$ at $4{ }^{\circ} \mathrm{C}$. The freeze-thaw stability for $3 \mathrm{cy}$ cles was in the range of $70.83-99.22 \%$. While His and HIST were more susceptible to degradation during sample preparation and instrumental analysis, BAs (CDCA, DCA, and LCA) were relatively susceptible to repeated freeze-thawing. Therefore, in this study, profiling of all gastric fluid samples was performed within $24 \mathrm{~h}$ after thawing. The matrix effects by use of 7 SIL internal standards were in the range of $93.50-113.92 \%$. These results showed that influences of residual derivatization reagents and interference materials in sample matrices were negligible. The validation results verified that the established method can provide reliable and accurate quantification data for HIST, His, and 5 BAs in gastric fluid samples.

\section{Application to human gastric fluid}

With this method, quantitative profiling of HIST, His, and BAs in human gastric fluid was performed. To precisely and accurately quantify and identify target analytes in gastric fluid samples, 7 SIL internal standards corresponding to all targets were employed and could compensate for signal distortion of analytes in each sample. The MRM chromatograms of 5 BAs, His, HIST, and their internal standards were reconstructed as shown in Fig. 5, and the individual MRM chromatograms showed that the analytes in human gastric fluid were sensitively and selectively detected without matrix interference. Concentration levels of target metabolites in gastric fluid were calculated by peak area ratios of targets to ISs. Calculated concentrations of all target analytes in human gastric fluid are summarized in Table 3. To the best of our knowledge, physiological levels of BAs and His in gastric fluid have not been reported all together, while concentrations of HIST in human gastric fluid from healthy subjects have been reported in the range of 0.9-4.2 (mean; 2.3 ) ng/mL (Haimart et al. 1985; Robert et al. 1983). Concentrations of HIST in gastric fluid from patients with CSG obtained with our method were higher than those of previous reports. It was speculated that HIST release in gastric fluid of patients with gastritis was more highly stimulated than in that of healthy subjects (Queiroz et al. 1991).

Moreover, it has been reported that the gastric BA concentrations are correlated with $H$. pylori infection (Kawai et al. 2001; Mathai et al. 1991). For instance, it was reported that since high BA concentrations in gastric fluid inhibit adherence and growth of $H$. pylori in early stages of infection, $H$. pylori positive patients had lower gastric BA levels than $H$. pylori negative patients (Kawai et al. 2001). On the other hand, it was reported that patients with $H$. pylori-associated gastritis can have high intragastric BA concentrations, because $H$. pylori infection may induce duodenogastric reflux, which is well recognized as a cause of gastritis and even gastric cancer (Ladas et al. 1996; Sobala et al. 1993). Interestingly, latter findings are consistent with our findings that gastric BA levels in CSG patients with $H$. pylori infection are significantly higher than that without $H$. pylori infection, except for LCA (Table 3). For LCA, it was speculated that since most of LCA cannot be recycled by enterohepatic circulation and is removed via defecation, gastric LCA concentrations did not have significant correlation between $H$. pylori positive and negative groups. In conclusion, this method is suitable to simultaneously determine HIST, His, and 5 free BAs in human gastric fluid samples.

\section{Conclusion}

In this study, a UHPLC-MS/MS method combined with serial derivatization to simultaneously profile HIST, His, 


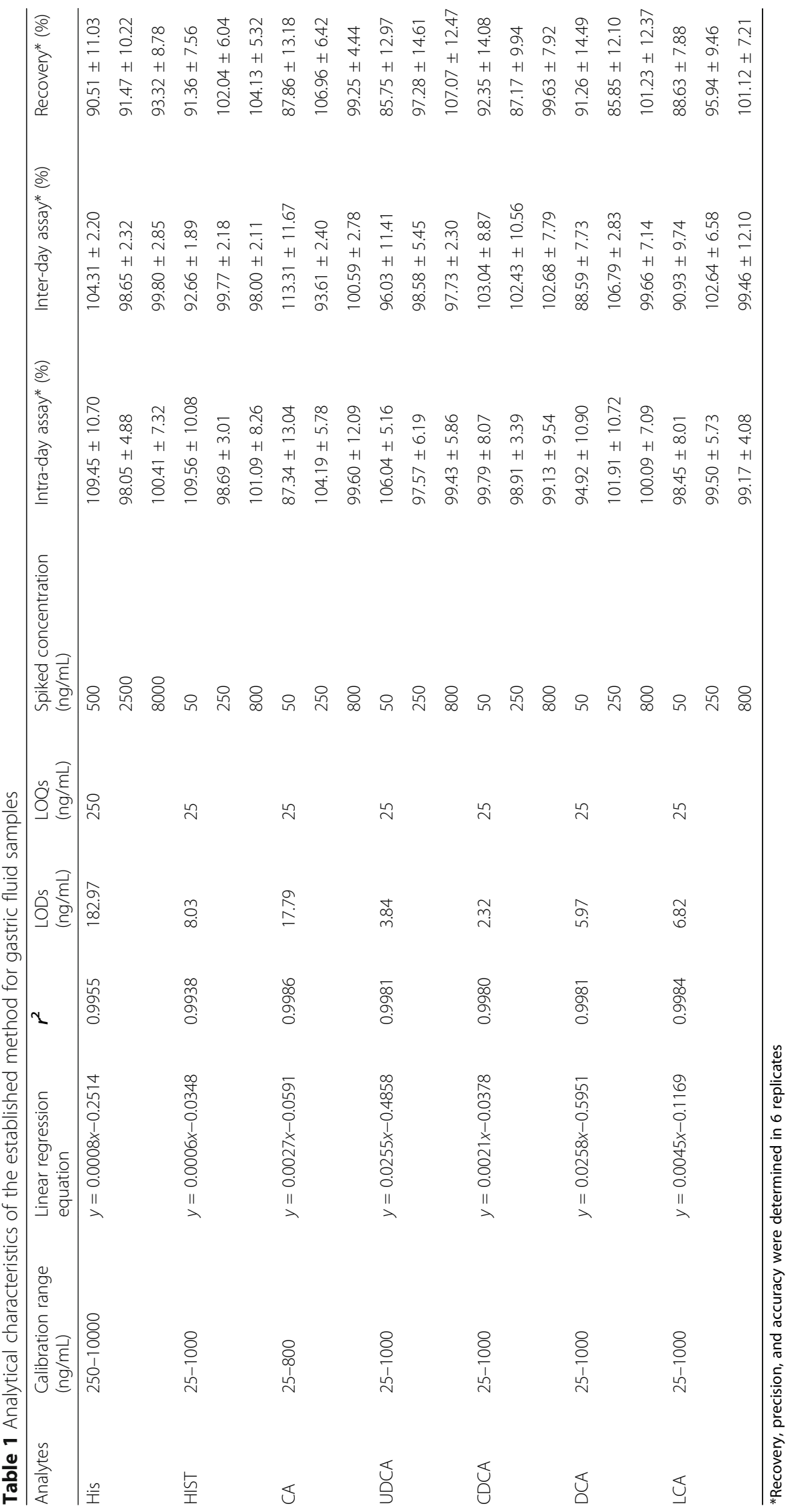


Table 2 Stability and matrix effect of HIST, His, and 5 BAs in spiked gastric fluid sample

\begin{tabular}{|c|c|c|c|c|c|c|c|c|c|}
\hline \multirow[t]{3}{*}{ Analytes } & \multirow{3}{*}{$\begin{array}{l}\text { Conc. } \\
\text { (ng/ } \\
\mathrm{mL})\end{array}$} & \multicolumn{7}{|l|}{ Stability* (\%) } & \multirow{3}{*}{$\begin{array}{l}\text { Matrix effect* } \\
(\%)\end{array}$} \\
\hline & & \multicolumn{3}{|c|}{ Room temperature } & \multicolumn{3}{|l|}{$4^{\circ} \mathrm{C}$} & \multirow{2}{*}{$\begin{array}{l}3 \text { freeze- } \\
\text { thaw cycle }\end{array}$} & \\
\hline & & $24 \mathrm{~h}$ & $48 \mathrm{~h}$ & $72 \mathrm{~h}$ & $24 \mathrm{~h}$ & $48 \mathrm{~h}$ & $72 \mathrm{~h}$ & & \\
\hline \multirow[t]{3}{*}{ His } & 250 & $86.38 \pm 5.42$ & $75.61 \pm 7.63$ & $74.06 \pm 3.65$ & $84.00 \pm 4.99$ & $81.05 \pm 6.71$ & $82.21 \pm 2.35$ & $96.32 \pm 4.68$ & $96.60 \pm 5.68$ \\
\hline & 500 & $78.13 \pm 0.84$ & $77.19 \pm 3.86$ & $77.71 \pm 4.97$ & $80.20 \pm 5.18$ & $77.55 \pm 4.29$ & $84.71 \pm 4.32$ & $91.82 \pm 3.69$ & \\
\hline & 2500 & $75.09 \pm 2.11$ & $82.36 \pm 3.69$ & $81.97 \pm 3.36$ & $85.96 \pm 5.79$ & $85.92 \pm 4.53$ & $87.85 \pm 6.08$ & $97.57 \pm 4.11$ & \\
\hline \multirow[t]{3}{*}{ HIST } & 25 & $89.07 \pm 4.09$ & $83.60 \pm 6.05$ & $87.31 \pm 3.25$ & $76.98 \pm 6.24$ & $86.51 \pm 2.78$ & $82.79 \pm 4.26$ & $97.49 \pm 1.87$ & $110.62 \pm 9.98$ \\
\hline & 50 & $76.82 \pm 2.91$ & $76.13 \pm 2.49$ & $80.55 \pm 3.72$ & $75.79 \pm 6.13$ & $82.68 \pm 1.76$ & $82.40 \pm 4.00$ & $89.14 \pm 5.43$ & \\
\hline & 250 & $79.60 \pm 4.57$ & $87.67 \pm 2.69$ & $89.50 \pm 1.52$ & $83.82 \pm 3.11$ & $91.10 \pm 1.07$ & $90.02 \pm 1.78$ & $98.31 \pm 2.39$ & \\
\hline \multirow[t]{3}{*}{ UDCA } & 25 & $94.89 \pm 3.47$ & $87.33 \pm 5.82$ & $88.31 \pm 1.72$ & $89.91 \pm 3.04$ & $96.95 \pm 3.44$ & $88.43 \pm 4.11$ & $99.22 \pm 5.23$ & $93.50 \pm 7.52$ \\
\hline & 50 & $82.35 \pm 1.91$ & $84.52 \pm 2.92$ & $84.54 \pm 2.45$ & $92.50 \pm 3.34$ & $91.04 \pm 1.72$ & $87.98 \pm 1.78$ & $84.03 \pm 8.11$ & \\
\hline & 250 & $86.26 \pm 1.90$ & $85.97 \pm 7.65$ & $91.48 \pm 1.27$ & $93.68 \pm 2.55$ & $97.69 \pm 2.33$ & $91.94 \pm 2.01$ & $87.18 \pm 5.47$ & \\
\hline \multirow[t]{3}{*}{ CA } & 25 & $96.08 \pm 5.76$ & $85.92 \pm 4.79$ & $87.62 \pm 4.87$ & $88.88 \pm 4.44$ & $90.85 \pm 4.44$ & $88.05 \pm 2.63$ & $98.29 \pm 5.47$ & $113.92 \pm 11.87$ \\
\hline & 50 & $83.21 \pm 4.03$ & $86.54 \pm 5.39$ & $88.81 \pm 0.78$ & $95.93 \pm 6.86$ & $88.62 \pm 3.82$ & $87.83 \pm 4.07$ & $88.17 \pm 5.68$ & \\
\hline & 250 & $84.75 \pm 1.06$ & $86.20 \pm 4.46$ & $91.26 \pm 2.32$ & $91.91 \pm 2.36$ & $91.77 \pm 2.00$ & $89.53 \pm 2.54$ & $95.27 \pm 3.48$ & \\
\hline \multirow[t]{3}{*}{ CDCA } & 25 & $95.78 \pm 3.83$ & $84.95 \pm 3.26$ & $91.83 \pm 3.14$ & $91.36 \pm 3.29$ & $101.14 \pm 4.88$ & $84.76 \pm 5.28$ & $75.61 \pm 7.91$ & $104.82 \pm 10.00$ \\
\hline & 50 & $87.57 \pm 3.66$ & $95.49 \pm 5.48$ & $92.60 \pm 7.03$ & $104.88 \pm 2.04$ & $94.61 \pm 5.82$ & $91.13 \pm 3.96$ & $70.77 \pm 10.45$ & \\
\hline & 250 & $94.50 \pm 2.36$ & $91.40 \pm 9.45$ & $103.28 \pm 2.14$ & $108.03 \pm 1.95$ & $107.31 \pm 1.46$ & $100.74 \pm 5.11$ & $70.42 \pm 6.12$ & \\
\hline \multirow[t]{3}{*}{ DCA } & 25 & $97.79 \pm 5.06$ & $92.93 \pm 1.43$ & $96.25 \pm 6.98$ & $96.85 \pm 5.17$ & $103.98 \pm 4.02$ & $91.67 \pm 3.48$ & $85.75 \pm 4.44$ & $104.15 \pm 10.44$ \\
\hline & 50 & $90.06 \pm 1.98$ & $97.35 \pm 3.94$ & $95.48 \pm 4.81$ & $107.31 \pm 3.66$ & $95.47 \pm 5.17$ & $91.59 \pm 2.95$ & $74.61 \pm 6.28$ & \\
\hline & 250 & $91.50 \pm 3.48$ & $97.22 \pm 9.01$ & $98.24 \pm 1.16$ & $105.35 \pm 1.59$ & $106.21 \pm 2.83$ & $95.09 \pm 4.32$ & $72.67 \pm 6.78$ & \\
\hline \multirow[t]{3}{*}{ LCA } & 25 & $98.08 \pm 3.26$ & $103.03 \pm 3.45$ & $104.43 \pm 2.37$ & $107.03 \pm 1.78$ & $105.87 \pm 3.71$ & $104.61 \pm 3.06$ & $87.37 \pm 1.46$ & $103.47 \pm 10.18$ \\
\hline & 50 & $93.41 \pm 5.14$ & $104.34 \pm 1.18$ & $104.08 \pm 2.49$ & $107.77 \pm 3.97$ & $107.37 \pm 3.10$ & $100.29 \pm 4.77$ & $76.51 \pm 6.28$ & \\
\hline & 250 & $104.65 \pm 3.50$ & $101.39 \pm 5.81$ & $105.73 \pm 7.50$ & $99.73 \pm 0.95$ & $109.64 \pm 3.94$ & $97.68 \pm 4.40$ & $70.83 \pm 9.21$ & \\
\hline
\end{tabular}

*Stabilities and matrix effects were determined in triplicate

and 5 BAs in gastric fluid from humans was described. The developed serial derivatization method enabled (1) one-pot derivatization of multifunctional groups on analytes with different physicochemical properties, (2) reasonable RPLC separation by increasing the hydrophobicity of highly polar compounds, and (3) sensitive and selective MRM detection using characteristic fragment ions and neutral losses. This UHPLC-MS/MS-MRM method provided sufficient detection sensitivity and selectivity to quantitatively profile trace levels of HIST, His, and BAs in biological samples. The investigated validation results of this method were within acceptable values. The established analytical method was successfully applied to gastric fluid from patients with CSG with

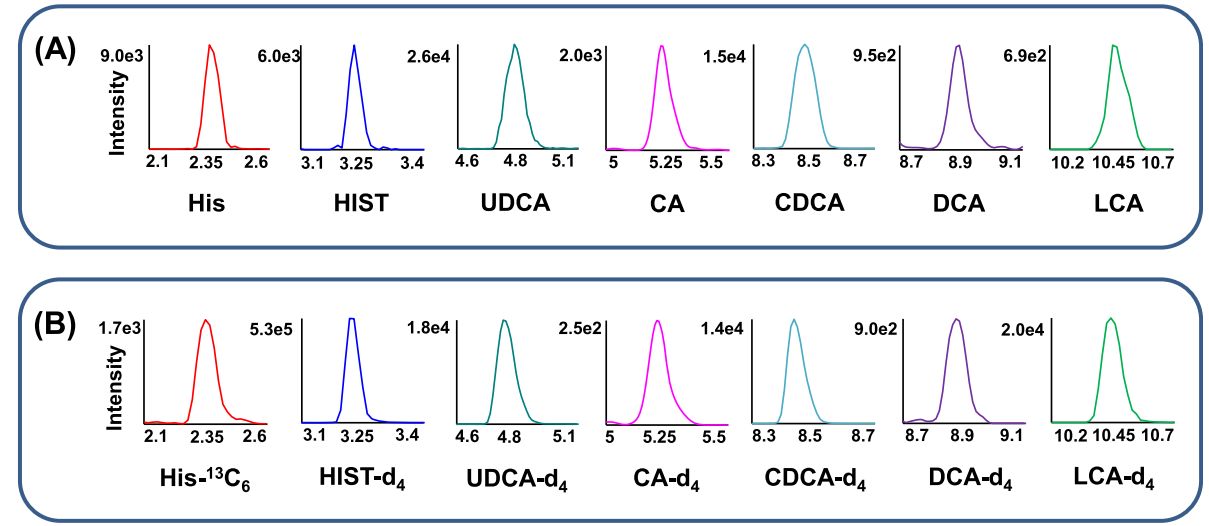

Fig. 5 MRM chromatograms of HIST, His, 5 BAs, and their corresponding internal standards in human gastric fluid 
Table 3 Concentrations of HIST, His, and 5 BAs in gastric fluid of patients with CSG with or without H. pylori infection

\begin{tabular}{llll}
\hline Compound name & CSG with H. pylori infection $(n=5)$ & CSG without H. pylori infection $(n=15)$ & $p$ value* \\
\hline His & $2479.68 \pm 1402.26$ & $2197.68 \pm 2573.37$ & 0.315 \\
HIST & $75.77 \pm 6.78$ & $75.35 \pm 7.16$ & 0.694 \\
CA & $43.11 \pm 8.08$ & $38.86 \pm 3.64$ & 0.026 \\
UDCA & $5057.53 \pm 8788.92$ & $878.84 \pm 2340.94$ & 0.032 \\
CDCA & $533.56 \pm 848.97$ & $68.83 \pm 91.20$ & 0.026 \\
DCA & $169.15 \pm 266.17$ & $43.59 \pm 33.41$ & 0.040 \\
LCA & $37.62 \pm 9.48$ & $33.06 \pm 1.98$ & 0.206 \\
\hline
\end{tabular}

(Mean \pm S.D. $\mathrm{ng} / \mathrm{mL}$ )

${ }^{*} P$ values were determined with a Mann-Whitney $U$ test

or without $H$. pylori infection. Therefore, this method is expected to be a useful tool to observe the metabolic alterations of HIST, His, and 5 BAs in biological samples and will be helpful for understanding the etiological mechanisms of gastric cancer and to identify potential biomarkers to diagnose gastric diseases in clinical applications.

\section{Supplementary information}

Supplementary information accompanies this paper at https://doi.org/10. 1186/s40543-020-00218-6.

Additional file 1: Supplementary Fig. 1. Serial derivatization

procedures to primary amine and carboxylate groups on HIST, His, and BAs. Supplementary Fig. 2. MRM chromatograms of HIST, His, and 5 BAs according to derivatization order: (A) DMED-Bz derivatization and (B) Bz-DMED derivatization. SupplementaryFig. 3. MRM chromatograms of HIST, His, 5 BAs, and their corresponding internal standards in SGF $(10 \mu \mathrm{g} / \mathrm{mL}$ for His and $1 \mu \mathrm{g} / \mathrm{mL}$ for HIST and 5 BAs) Supplementary Table 1. MRM transitions and parameters. Supplementary Table S2. Derivatization yields for HIST, His, and BAs.

\section{Abbreviations}

BA: Bile acid; BzCl: Benzoyl chloride; CA: Cholic acid;

CDCA: Chenodeoxycholic acid; CSG: Chronic superficial gastritis;

DCA: Deoxycholic acid; DMED: N,N-Dimethylethylenediamine; FXR: Farnesoid X receptor; HDC: Histidine decraboxylase; His: Histidine; HIST: Histamine; LCA: Lithocholic acid; MRM: Multiple reaction monitoring; MS/MS: Tandem mass spectrometry; UDCA: Ursodeoxycholic acid; UHPLC: Ultra-high performance liquid chromatography

\section{Acknowledgements}

Not applicable

\section{Authors' contributions}

All authors read and approved the final manuscript.

\section{Funding}

This study was financially supported by the National Research Foundation of Korea (2017M3A9F3047858) and the KIST Institutional Program (2Z05790-19032).

\section{Availability of data and materials}

The datasets of this manuscript are available upon request.

\section{Competing interests}

There are no conflicts of interest to declare.

\section{Author details}

${ }^{1}$ College of Pharmacy, Kyung Hee University, Seoul 02447, Republic of Korea. ${ }^{2}$ Department of Internal Medicine, Institute of Gastroenterology, Yonsei University College of Medicine, Seoul 03722, Republic of Korea. ${ }^{3}$ Molecular Recognition Research Center, Korea Institute of Science and Technology, Seoul 02792, Republic of Korea.

Received: 6 January 2020 Accepted: 15 May 2020

Published online: 03 June 2020

\section{References}

Annesley TM. Ion suppression in mass spectrometry. Clin Chem. 2003;49(7):10414.

Bernstein H, Bernstein C, Payne CM, Dvorak K. Bile acids as endogenous etiologic agents in gastrointestinal cancer. World J Gastroenterol. 2009;15(27):3329-40.

Choi JM, Park WS, Song KY, Lee HJ, Jung BH. Development of simultaneous analysis of tryptophan metabolites in serum and gastric juice - an investigation towards establishing a biomarker test for gastric cancer diagnosis. Biomed Chromatogr. 2016;30:1963-74.

Dams R, Huestis MA, Lambert WE, Murphy CM. Matrix effect in bio-analysis of illicit drugs with LC-MS/MS: influence of ionization type, sample preparation, and biofluid. J Am Soc Mass Spectrom. 2003;14:1290-4.

Deng K, Lin S, Zhou L, Geng Q, Li Y, Xu M, et al. Three aromatic amino acids in gastric juice as potential biomarkers for gastric malignancies. Anal Chim Acta Elsevier BV. 2011;694:100-7.

Deng K, Lin S, Zhou L, Li Y, Chen M, Wang Y, et al. High levels of aromatic amino acids in gastric juice during the early stages of gastric cancer progression. PLoS One. 2012;7(11):e49434.

Falus A, Pós Z, Darvas Z. Histamine in normal and malignant cell proliferation. Adv Exp Med Biol. 2011;709:109-23.

Gadaleta RM, Cariello M, Sabbà C, Moschetta A. Tissue-specific actions of FXR in metabolism and cancer. Biochim Biophys Acta Mol Cell Biol Lipids. 2015; 1851(1):30-9.

Gao X, Pujos-Guillot E, Martin J-F, Galan P, Juste C, Jia W, et al. Metabolite analysis of human fecal water by gas chromatography/mass spectrometry with ethyl chloroformate derivatization. Anal Biochem. 2009;393(2):163-75.

García-Cañaveras JC, Donato MT, Castell JV, Lahoz A. Targeted profiling of circulating and hepatic bile acids in human, mouse, and rat using a UPLCMRM-MS-validated method. J. Lipid Res. 2012;53:2231-41.

Gornischeff A, Liigand J, Rebane R. A systematic approach toward comparing electrospray ionization efficiencies of derivatized and non-derivatized amino acids and biogenic amines. J Mass Spectrom. 2018;53(10):997-1004.

Gropper SS, Smith JL, Groff JL. Advanced nutrition and human metabolism. Fifth Edit. Belmont: Wadsworth; 2005.

Haimart M, Launay JM, Zürcher G, Cauet N, Dreux C, Da Prada M. Simultaneous determination of histamine and Na-methylhistamine in biological samples by an improved enzymatic single isotope assay. Agents Actions. 1985;16(34):71-5.

He Y, Luo Y, Hou H, Chen H, Chen J, Fu Y, et al. Profiling of carboxyl-containing metabolites in smokers and non-smokers by stable isotope labeling combined with LC-MS/MS. Anal Biochem. 2019;569:1-9.

Huang T, Armbruster M, Lee R, Hui DS, Edwards JL. Metabolomic analysis of mammalian cells and human tissue through one-pot two stage 
derivatizations using sheathless capillary electrophoresis-electrospray ionization-mass spectrometry. J Chromatogr A. 2018a;1567:219-25.

Huang T, Armbruster MR, Coulton JB, Edwards JL. Chemical tagging in mass spectrometry for systems biology. Anal Chem. 2019;91(1):109-25.

Huang T, Toro M, Lee R, Hui DS, Edwards JL. Multi-functional derivatization of amine, hydroxyl, and carboxylate groups for metabolomic investigations of human tissue by electrospray ionization mass spectrometry. Analyst Royal Soc Chem. 2018b;143(14):3408-14.

Kawai Y, Tazuma S, Inoue M. Bile acid reflux and possible inhibition of Helicobacter pylori infection in subjects without gastric surgery. Dig Dis Sci. 2001;46(8):1779-83.

Ku HJ, Kim HY, Kim HH, Park HJ, Cheong JH. Bile acid increases expression of the histamine-producing enzyme, histidine decarboxylase, in gastric cells. World J Gastroenterol. 2014;20(1):175-82.

Ladas SD, Katsogridakis J, Malamou H, Giannopoulou H, Kesse-Elia M, Raptis SA. Helicobacter pylori may induce bile reflux: link between $\mathrm{H}$ pylori and bile induced injury to gastric epithelium. Gut. 1996;38(1):15-8.

Lee W, Park NH, Ahn T, Chung BC, Hong J. Profiling of a wide range of neurochemicals in human urine by very-high-performance liquid chromatography-tandem mass spectrometry combined with in situ selective derivatization. J Chromatogr A. 2017;1526:47-57.

Lee W, Um J, Hwang B, Lee YC, Chung BC, Hong J. Assessing the progression of gastric cancer via profiling of histamine, histidine, and bile acids in gastric juice using LC-MS/MS. J Steroid Biochem Mol Biol. 2020;197:105539.

Liu J, Lin S, Li Z, Zhou L, Xue Y, Yan X, et al. Original Article A novel gastric juice index model for detecting early gastric cancer. Int J Clin Exp Med. 2017; 10(10):14425-35.

Liu J, Lin S, Li Z, Zhou L, Yan X, Xue Y, et al. Free amino acid profiling of gastric juice as a method for discovering potential biomarkers of early gastric cancer. Int J Clin Exp Pathol. 2018;11(5):2323-36.

Lu H, Yu J, Wang J, Wu L, Xiao H, Gao R. Simultaneous quantification of neuroactive dopamine serotonin and kynurenine pathway metabolites in gender-specific youth urine by ultra performance liquid chromatography tandem high resolution mass spectrometry. J Pharm Biomed Anal. 2016;122:42-51.

Lu PJ, Hsu PI, Chen CH, Hsiao M, Chang WC, Tseng HH, et al. Gastric juice acidity in upper gastrointestinal diseases. World J Gastroenterol. 2010;16(43):5496-501.

Makishima M, Okamoto AY, Repa JJ, Tu H, Learned RM, Luk A, et al. Identification of a nuclear receptor for bile acids. Science. 1999;284(5418):1362-5.

Malec PA, Oteri M, Inferrera V, Cacciola F, Mondello L, Kennedy RT. Determination of amines and phenolic acids in wine with benzoyl chloride derivatization and liquid chromatography-mass spectrometry. J Chromatogr A. 2017;1523: 248-56.

Mathai E, Arora A, Cafferkey M, Keane CT, O'Morain C. The effect of bile acids on the growth and adherence of Helicobacter pylori. Aliment Pharmacol Ther. 1991;5:653-68

Parks DJ, Blanchard SG, Bledsoe RK, Chandra G, Consler TG, Kliewer SA, et al. Bile acids: Natural ligands for an orphan nuclear receptor. Science. 1999; 284(5418):1365-8.

Queiroz DMM, Mendes EN, Rocha GA, Barbosa AJA, Carvalho AST, Cunha-Melo JR. Histamine concentration of gastric mucosa in Helicobacter pylori positive and negative children. Gut. 1991;32(5):464-6.

Robert JC, Vatier J, Nguyen Phuoc BK, Bonfils S. Determination of histamine, methylhistamines and histamine-o-phthaldialdehyde complexes by two high-performance liquid chromatographic procedures. J Chromatogr B. 1983; 273:275-87.

Smith $J$. The role of gastric acid in preventing foodborne disease and how bacteria overcome acid conditions. J Food Prot. 2003;66(7):1292-303.

Sobala GM, Oconnor HJ, Dewar EP, King RFG, Axon ATR, Dixon MF. Bile reflux and intestinal metaplasia in gastric-mucosa. J Clin Pathol. 1993;46(3):235-40.

Song P, Mabrouk OS, Hershey ND, Kennedy RT. In vivo neurochemical monitoring using benzoyl chloride derivatization and liquid chromatography -mass spectrometry. Anal Chem. 2012;84(1):412-9.

Tufi S, Lamoree M, de Boer J, Leonards P, de Boer J, Leonards P, et al. Simultaneous analysis of multiple neurotransmitters by hydrophilic interaction liquid chromatography coupled to tandem mass spectrometry. Chromatogr A. 2015;1395:79-87.

U.S. Department of Health and Human Services Food and Drug Administration. Bioanalytical Method Validation: Guidance for Industry. 2018.
Wan Y-JY, Sheng L. Regulation of bile acid receptor activity. Liver Res. 2018;2(4): 180-5.

Wong J-MT, Malec PA, Mabrouk OS, Ro J, Dus M, Kennedy RT. Benzoyl chloride derivatization with liquid chromatography-mass spectrometry for targeted metabolomics of neurochemicals in biological samples. J Chromatogr A. 2016;1446:78-90

Yin S, Su M, Xie G, Li X, Wei R, Liu C, et al. Factors affecting separation and detection of bile acids by liquid chromatography coupled with mass spectrometry in negative mode. Anal Bioanal Chem. 2017;409(23):5533-45.

Yuan B-F, Zhu Q-F, Guo N, Zheng S-J, Wang Y-L, Wang J, et al. Comprehensive profiling of fecal metabolome of mice by integrated chemical isotope labeling-mass spectrometry analysis. Anal Chem. 2018;90(5):3512-20.

Zheng X, Kang A, Dai C, Liang Y, Xie T, Xie L, et al. Quantitative analysis of neurochemical panel in rat brain and plasma by liquid chromatography -tandem mass spectrometry. Anal Chem. 2012;84(22):10044-51.

Zhu Q-F, Hao Y-H, Liu M-Z, Yue J, Ni J, Yuan B-F, et al. Analysis of cytochrome P450 metabolites of arachidonic acid by stable isotope probe labeling coupled with ultra high-performance liquid chromatography/mass spectrometry. J Chromatogr A. 2015;1410:154-63.

Zhu Q-F, Zhang Z, Liu P, Zheng S-J, Peng K, Deng Q-Y, et al. Analysis of liposoluble carboxylic acids metabolome in human serum by stable isotope labeling coupled with liquid chromatography-mass spectrometry. J Chromatogr A. 2016;1460:100-9.

\section{Publisher's Note}

Springer Nature remains neutral with regard to jurisdictional claims in published maps and institutional affiliations.

\section{Submit your manuscript to a SpringerOpen ${ }^{\circ}$ journal and benefit from:}

- Convenient online submission

- Rigorous peer review

- Open access: articles freely available online

High visibility within the field

- Retaining the copyright to your article

Submit your next manuscript at $\boldsymbol{\nabla}$ springeropen.com 